\title{
Reduced expression of claudin-7 is associated with poor outcome in non-small cell lung cancer
}

\author{
TAKETSUGU YAMAMOTO ${ }^{1}$, TAKASHI OSHIMA ${ }^{2}$, KAZUE YOSHIHARA $^{2}$, SUMITAKA YAMANAKA $^{3}$, \\ TEPPEI NISHII ${ }^{3}$, HIROMASA ARAI $^{3}$, KENJI INUI $^{3}$, TAKESHI KANEKO ${ }^{3}$, AKINORI NOZAWA $^{4}$, \\ TETSUKAN WOO $^{1}$, YASUSHI RINO ${ }^{1}$, MUNETAKA MASUDA ${ }^{1}$ and TOSHIO IMADA ${ }^{1}$
}

${ }^{1}$ Department of Surgery, Yokohama City University, Kanagawa $236-0004 ;{ }^{2}$ Gastroenterological Center; ${ }^{3}$ Respiratory Center; ${ }^{4}$ Department of Pathology, Yokohama City University Medical Center, Yokohama-city, Kanagawa 232-0024, Japan

Received December 29, 2009; Accepted March 2, 2010

DOI: 10.3892/ol_00000088

\begin{abstract}
Claudin-7 is a tight junction protein that plays an important role in tumorigenesis, tumor invasion and metastasis. We examined the clinical significance of claudin-7 expression in 75 postsurgical non-small cell lung cancer (NSCLC) patients. Claudin-7 expression was measured immunohistochemically and was found to be high in 25 patients $(33.3 \%)$ and low in 50 (66.7\%). Survival was significantly poorer in patients with claudin-7-low than in those with claudin-7-high NSCLCs $(\mathrm{P}=0.024)$. In particular, survival was significantly poorer in patients with claudin-7-low than in those with claudin-7-high squamous cell carcinomas $(\mathrm{P}=0.011)$. A reduced expression of claudin-7 was associated with poor outcome in NSCLCs. Claudin-7 may thus be a useful biomarker and a potential therapeutic target in patients with NSCLC.
\end{abstract}

\section{Introduction}

Primary lung cancer is the leading cause of cancer-related mortality in Japan and in Western countries, with non-small cell lung cancer (NSCLC) accounting for approximately $80 \%$ of primary lung cancers $(1,2)$. Surgical resection is the only potentially curative treatment in patients with early disease. However, 5-year survival after surgical resection remains unsatisfactory, ranging from $73 \%$ in stage IA disease to $25 \%$ in stage IIIA disease. Improvement in the poor survival of patients with NSCLC requires better clinical predictors of outcomes and of response to specific therapeutic interventions.

Cell-to-cell adhesion is generally reduced in various human cancer types. The dissociation of cancer nests is a crucial step in metastasis. The suppression of cell-to-cell adhesion may trigger the release of cancer cells from primary

Correspondence to: Dr Taketsugu Yamamoto, Department of Surgery, Yokohama City University, 3-9 Fukuura, Kanazawa-ku, Yokohama-city, Kanagawa 236-0004, Japan

E-mail: taketugu@yokohama-cu.ac.jp

Key words: claudin-7, prognostic factor, non-small cell lung cancer cancer nests, increasing tumor invasion (3). Tight junctions are cell-cell junctions located at the apical end of the lateral membrane surface of epithelial or endothelial cells. Tight junctions consist of membrane and peripheral proteins. Occludin and claudins are membrane proteins, and zonula occludens (ZO)-1, ZO-2 and ZO-3 are peripheral proteins. Claudin-7 is a member of the 24-claudin multigene family, associated with cancer. Loss of claudin-7 expression has been confirmed in ductal carcinoma of the breast and squamous cell carcinoma of the head and neck $(4,5)$. Usami et al reported that a reduced claudin-7 expression correlates with tumor invasion and metastasis in squamous cell carcinoma of the esophagus (6). Whether the expression of claudin-7 is associated with the malignant potential of NSCLC remains to be clarified. The present study evaluated the clinical significance of claudin-7, especially its relation to outcome, in resected NSCLC.

\section{Materials and methods}

We retrospectively reviewed a total of 76 consecutive patients with pathological (p)-stage I to III NSCLC. The patients underwent complete tumor resection and nodal dissection without any pre-operative therapy at the Respiratory Center, Yokohama City University Medical Center between January 1st, 2000 and November 30th, 2003. One patient succumbed to operation-related causes and was excluded from the study. The study group thus comprised 75 patients: 45 with adenocarcinomas, 25 with squamous cell carcinomas and 5 patients with large cell carcinomas. The mean follow-up was 1,466 days (range 106-3,328). Informed consent was obtained from each patient. This study was approved by Ethics Committee of the Yokohama City Medical Center.

Immunohistochemistry. The primary antibody used to detect claudin-7 was a mouse monoclonal antibody (Zymed, San Francisco, CA, USA). Formalin-fixed, paraffin-embedded tissue specimens were cut into $4-\mu \mathrm{m}$ sections and mounted on slides. The sections were deparaffinized and rehydrated. The slides were then heated in a microwave oven three times for $5 \mathrm{~min}$ each in a $10-\mu \mathrm{mol} / 1$ citrate buffer solution at $\mathrm{pH} 6.0$ and cooled at room temperature for $20 \mathrm{~min}$. After quenching the endogenous peroxidase activity with $3 \% \mathrm{H}_{2} \mathrm{O}_{2}$ for $5 \mathrm{~min}$, the 

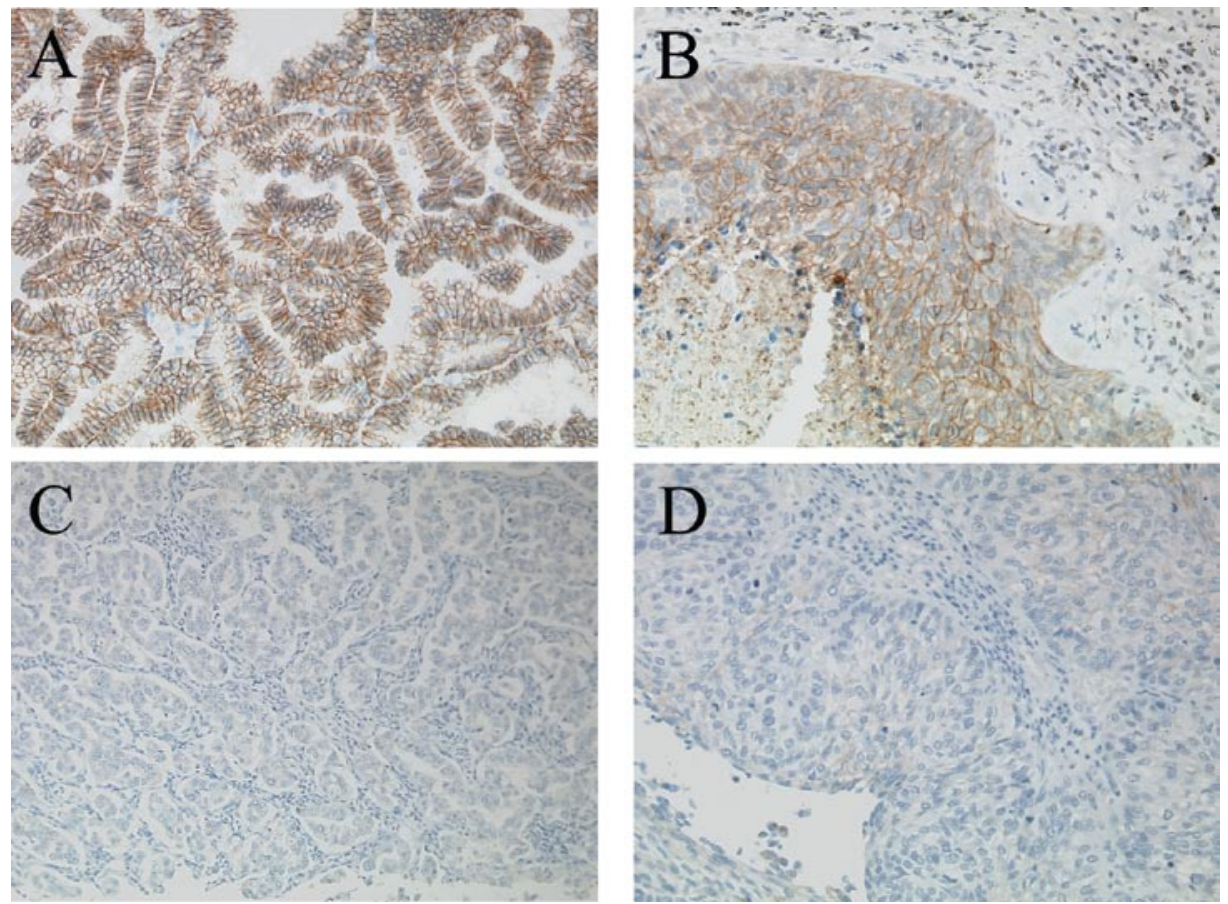

Figure 1. Immunostaining of NSCLC in serial sections. An adenocarcinoma with high expression of claudin-7 (A) and a squamous cell carcinoma with high expression of claudin-7 (B). An adenocarcinoma with low expression of claudin-7 (C) and a squamous cell carcinoma with low expression of claudin-7 (D).

sections were incubated for $60 \mathrm{~min}$ at room temperature, with the primary antibody diluted at 1:100. Endogenous biotin was blocked by the Dako Biotin blocking system (Dako, Glostrup, Denmark), according to the manufacturer's specifications. After rinsing, specific staining was visualized with the use of an LSAB+ system-HRP system (Dako). Color was produced by the application of 3,3'-diaminobenzidine for $10 \mathrm{~min}$. The sections were counterstained with Meyer's hematoxylin (Muto Pure Chemicals, Tokyo, Japan).

Samples were scored semiquantitatively and qualitatively for every section, without knowledge of the clinical data. The distribution of stained cells was scored as follows: 1, 0-30\%; $2,31-60 \%$ and $3,61-100 \%$. The intensity of claudin-7 expression was scored from 1 to 3 as follows: grade 1, weak staining; grade 2 , moderate staining and grade 3 , strong staining. Strong staining of $\geq 61 \%$ cells (i.e., both distribution score and intensity score $=3$ ) was classified as claudin-7-high. The remaining staining patterns were classified as claudin-7-low.

Statistical analysis. Counts were compared by the $\chi^{2}$ test. Continuous data were compared using Student's t-test. The postoperative survival rate was analyzed by the KaplanMeier method and differences in survival rates were assessed using the log-rank test. Death from any cause was included in the calculation of postoperative survival. Differences were considered significant when $\mathrm{P}<0.05$. Statistical manipulations were performed using the SPSS for Windows software system (SPSS, Inc., Chicago, IL, USA).

\section{Results}

The subjects included were 52 men and 23 women with a mean age of 65.2 years (range 32-82; median 65). The most common histological type of tumor was adenocarcinoma
Table I. Immunohistochemical results for claudin-7 in NSCLC.

\begin{tabular}{lrcr}
\hline Distribution (\%) & \multicolumn{3}{c}{ Intensity (\%) } \\
\cline { 2 - 4 } & \multicolumn{1}{c}{1} & \multicolumn{1}{c}{3} \\
\hline $1(0-30)$ & $28(37.3)$ & $1(1.3)$ & $0(0.0)$ \\
$2(31-60)$ & $8(10.6)$ & $3(4.0)$ & $0(0.0)$ \\
$3(61-100)$ & $3(4.0)$ & $7(9.3)$ & $25(33.3)$
\end{tabular}

(60.0\%; 45 cases), followed by squamous cell carcinoma (33.3\%; 25 cases) and large cell carcinoma (6.7\%; 5 cases). Tumor size was $\mathrm{T} 1$ in 34 patients (45.3\%), T2 in 28 (37.3\%), $\mathrm{T} 3$ in $9(12.0 \%)$ and T4 in $4(5.3 \%)$. Thirty-six patients $(48.0 \%)$ had no metastasis to regional lymph nodes (N0), whereas 11 (14.7\%) had metastatic involvement of the hilar lymph nodes (N1) and 28 (37.3\%) had metastases to the mediastinal nodes (N2, N3). Thirty tumors $(40.0 \%)$ were classified as stage I, $15(20.0 \%)$ were stage II and $30(40.0 \%)$ were stage III. At the end of follow-up, 34 patients $(45.3 \%)$ were alive and 41 (54.7\%) had succumbed to the disease.

The immunohistochemical expression of claudin-7 was detected in the epithelial cell membranes of the normal bronchial mucosa. Claudin-7 expression was also observed in lung cancer cells (Fig. 1). The immunohistochemical distribution and intensity of claudin-7 expression are summarized in Table I. Among the 75 carcinomas studied, 25 (33.3\%) strongly expressed claudin- 7 . In the 75 carcinomas, $\geq 61 \%$ of the tumor cells stained positively. Therefore, 25 carcinomas were classified as claudin-7-high and 50 (66.7\%) were classified as claudin-7-low. 
Table II. Patient characteristics and the claudin-7 expression status.

\begin{tabular}{|c|c|c|c|c|}
\hline & \multirow[t]{2}{*}{ No. of patients (\%) } & \multicolumn{2}{|c|}{ Claudin-7 expression } & \multirow[t]{2}{*}{$\mathrm{P}$-value } \\
\hline & & High $(\%)$ & Low $(\%)$ & \\
\hline All patients & $75(100)$ & $25(33.3)$ & $50(66.7)$ & \\
\hline Age $($ mean $\pm \mathrm{SD})$ & $65.2 \pm 9.49$ & $65.8 \pm 6.98$ & $64.9 \pm 10.5$ & 0.702 \\
\hline \multicolumn{5}{|l|}{ Gender } \\
\hline Male & $52(69.3)$ & $15(28.8)$ & $37(71.2)$ & \multirow[t]{2}{*}{0.250} \\
\hline Female & $23(30.7)$ & $10(43.5)$ & $13(56.5)$ & \\
\hline \multicolumn{5}{|l|}{ Histological type } \\
\hline Adenocarcinoma & $45(60.0)$ & $16(35.6)$ & $29(64.4)$ & \multirow[t]{3}{*}{0.262} \\
\hline Squamous cell carcinoma & $25(33.3)$ & $6(24.0)$ & $19(76.0)$ & \\
\hline Large cell carcinoma & $5(6.7)$ & $3(60.0)$ & $2(40.0)$ & \\
\hline \multicolumn{5}{|l|}{ P-stage } \\
\hline I & $30(40.0)$ & $12(40.0)$ & $18(60.0)$ & \multirow[t]{3}{*}{0.178} \\
\hline II & $15(20.0)$ & $2(13.3)$ & $13(86.7)$ & \\
\hline III & $30(40.0)$ & $11(36.7)$ & $19(63.3)$ & \\
\hline \multicolumn{5}{|l|}{ Smoking } \\
\hline Non-smoker & $20(26.7)$ & $7(35.0)$ & $13(65.0)$ & \multirow[t]{2}{*}{0.854} \\
\hline Smoker & $55(73.3)$ & $18(32.7)$ & $37(67.3)$ & \\
\hline \multicolumn{5}{|l|}{ T-factor } \\
\hline $\mathrm{T} 1$ & $34(45.3)$ & $12(35.3)$ & $22(64.7)$ & \multirow[t]{4}{*}{0.452} \\
\hline $\mathrm{T} 2$ & $28(37.3)$ & $10(35.7)$ & $18(64.3)$ & \\
\hline $\mathrm{T} 3$ & $9(12.0)$ & $1(11.1)$ & $8(88.9)$ & \\
\hline $\mathrm{T} 4$ & $4(5.3)$ & $2(50.0)$ & $2(50.0)$ & \\
\hline \multicolumn{5}{|l|}{$\mathrm{N}$-factor } \\
\hline N0 & $36(48.0)$ & $13(36.1)$ & $23(63.9)$ & \multirow[t]{4}{*}{0.353} \\
\hline N1 & $11(14.7)$ & $2(18.2)$ & $9(81.8)$ & \\
\hline $\mathrm{N} 2$ & $27(36.0)$ & $9(33.3)$ & $18(66.7)$ & \\
\hline N3 & $1(1.3)$ & $1(100)$ & $0(0.0)$ & \\
\hline
\end{tabular}

Table III. Claudin-7 expression and postoperative survival in NSCLC.

\begin{tabular}{|c|c|c|c|}
\hline & \multicolumn{2}{|c|}{ 5-year survival rate $(\%)$} & \multirow[b]{2}{*}{ P-value } \\
\hline & Claudin-7 high patients & Claudin-7 low patients & \\
\hline All patients & 61.3 & 41.4 & 0.024 \\
\hline \multicolumn{4}{|l|}{ Gender } \\
\hline Male & 57.7 & 32.4 & 0.035 \\
\hline Female & 66.6 & 68.3 & 0.855 \\
\hline \multicolumn{4}{|l|}{ Histological type } \\
\hline Adenocarcinoma & 53.5 & 54.4 & 0.664 \\
\hline Squamous cell carcinoma & 80.0 & 26.3 & 0.011 \\
\hline \multicolumn{4}{|l|}{ P-stage } \\
\hline $\mathrm{I}$ & 91.6 & 50.0 & 0.023 \\
\hline II, III & 38.5 & 36.4 & 0.365 \\
\hline \multicolumn{4}{|l|}{ Smoking } \\
\hline Non-smoker & 50.0 & 61.5 & 0.890 \\
\hline Smoker & 65.1 & 34.3 & 0.010 \\
\hline
\end{tabular}


$\mathbf{A}$

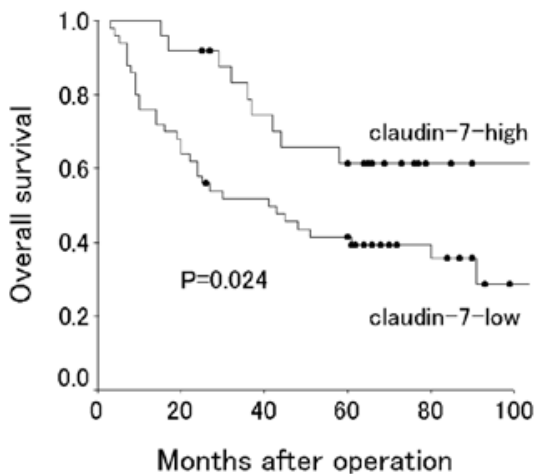

C

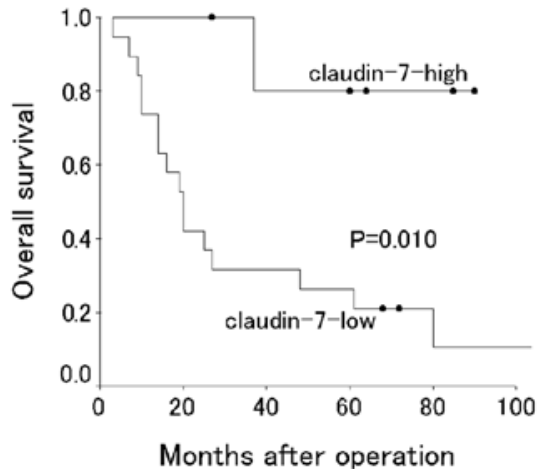

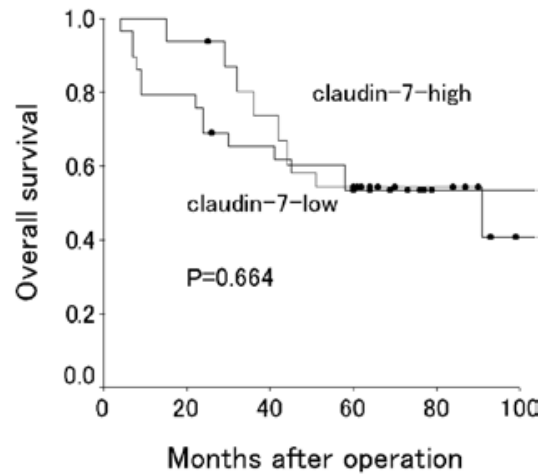

D

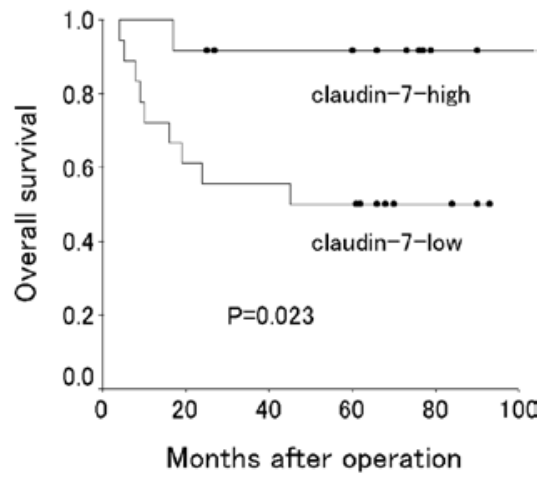

Figure 2. Overall survival of 75 patients with NSCLC according to claudin-7 status: (A) NSCLCs, (B) adenocarcinomas, (C) squamous cell carcinomas and (D) stage I NSCLCs.

Claudin-7 expression status did not significantly correlate with any of the patient characteristics (Table II). The 5-year survival rate was $41.4 \%$ in patients with claudin-7-low tumors, compared to $61.3 \%$ in those with claudin-7-high tumors. Survival was significantly poorer in patients with claudin-7-low tumors than in those with claudin-7-high tumors $(\mathrm{P}=0.024)$ (Table III). Subsequently, we conducted subset analyses to investigate the prognostic significance of the claudin-7 status. Among patients with squamous cell carcinoma, the 5-year survival rate was significantly lower in claudin-7-low (26.3\%) than in claudin-7-high patients $(80.0 \%, \mathrm{P}=0.011)$. For patients with adenocarcinoma, survival was similar in claudin-7-high and claudin-7-low patients (5-year survival rate, 53.5 and $54.4 \%$, respectively; $\mathrm{P}=0.664$ ). Claudin-7-high was associated with a better 5-year survival than claudin-7-low in patients with stage I disease, male patients and smokers $(\mathrm{P}=0.023$, 0.035 and 0.010 , respectively) (Table III).

\section{Discussion}

Claudin-7 expression was reported in various malignant neoplasms. Kominsky et al showed that loss of the claudin-7 expression is associated with nodal metastasis in primary breast carcinomas (5). Sauer et al found that a reduced claudin-7 expression correlates with metastatic disease in breast carcinoma (7), while Usami et al reported that this correlates with tumor invasion and metastasis in squamous cell carcinoma of the esophagus (6). A reduced claudin-7 expression correlates with a high tumor grade in prostatic adenocarcinoma (8). Oshima et al reported that a reduced claudin-7 expression correlates with venous invasion and liver metastasis in colorectal cancer (9). In contrast, an increased claudin-7 expression was reported in various cancer types, such as intestinal-type gastric adenocarcinomas (10), ovarian cancer (11) and cervical carcinoma (12). On the other hand, Bello et al reported that a high and low immunoreactivity of claudin-7 is associated with a slightly poorer survival than median immunoreactivity in squamous cell carcinoma of the tongue (13). However, claudin-7 expression in NSCLC remains largely uninvestigated.

The present study assessed the immunohistochemical expression of claudin-7 in NSCLC. Two previous studies examined the immunohistochemical expression of claudin-7 in NSCLC. Moldvay et al reported a very strong expression of claudin-7 in almost all types of NSCLC, except for neuroendocrine tumors (14). Soini also reported a strong expression of claudin-7 in almost all NSCLCs studied (15). However, neither of these studies assessed the clinical significance of claudin-7 expression. We found that $66.7 \%$ of the NSCLCs showed a low claudin-7 expression, and two large cell neuroendocrine carcinomas showed a high claudin-7 expression. The discrepancies noted in the findings of previous studies may be related to differences in staining procedures, such as the antibody used.

To the best of our knowledge, this is the first study to examine the clinical significance of claudin-7 expression in NSCLC. We found that a reduced expression of claudin-7 was a significant predictor of poor outcome, especially in squamous 
cell carcinoma. In contrast to patients with squamous cell carcinoma, claudin-7 expression status was unrelated to the postoperative survival in patients with adenocarcinoma.

Among patients with stage I disease, survival was significantly poorer in those with claudin-7-low than in those with claudin-7-high tumors. This finding suggests that claudin-7 is a prognostic marker for early stage cancer. Although in our study claudin-7 expression was unrelated to patient characteristics, such as p-stage and nodal status, further experimental and clinical studies should be conducted to define the exact role of claudin-7 in the progression of NSCLC.

In conclusion, a reduced claudin-7 expression was associated with poor outcome in patients with NSCLC, particularly in squamous cell carcinoma, stage I disease, male gender and smokers. These results suggest that claudin-7 expression is a useful biomarker and a potential therapeutic target in patients with NSCLC.

\section{References}

1. Jemal A, Siegel R, Ward E, Murray T, Xu J and Thun MJ: Cancer statistics. CA Cancer J Clin 57: 43-66, 2007.

2. Cersosimo RJ: Lung cancer: a review. Am J Health Syst Pharm 59: 611-642, 2002.

3. Hirohashi S: Inactivation of the E-cadherin-mediated cell adhesion system in human cancers. Am J Pathol 153: 333-339, 1998.

4. Al Moustafa AE, Alaoui-Jamali MA, Batist G, et al: Identification of genes associated with head and neck carcinogenesis by cDNA microarray comparison between matched primary normal epithelial and squamous carcinoma cells. Oncogene 21 : 2634-2640, 2002.
5. Kominsky SL, Argani P, Korz D, et al: Loss of the tight junction protein claudin-7 correlates with histological grade in both ductal carcinoma in situ and invasive ductal carcinoma of the breast. Oncogene 22: 2021-2033, 2003.

6. Usami Y, Chiba H, Nakayama F, et al: Reduced expression of claudin-7 correlates with invasion and metastasis in squamous cell carcinoma of the esophagus. Hum Pathol 37: 569-577, 2006.

7. Sauer T, Pedersen MK, Ebeltoft K and Naess O: Reduced expression of Claudin-7 in fine needle aspirates from breast carcinomas correlate with grading and metastatic disease. Cytopathology 16: 193-198, 2005

8. Sheehan GM, Kallakury BV, Sheehan CE, Fisher HA, Kaufman RP Jr and Ross JS: Loss of claudins-1 and -7 and expression of claudins-3 and -4 correlate with prognostic variables in prostatic adenocarcinomas. Hum Pathol 38: 564-569, 2007.

9. Oshima T, Kunisaki C, Yoshihara K, et al: Reduced expression of the claudin-7 gene correlates with venous invasion and liver metastasis in colorectal cancer. Oncol Rep 19: 953-959, 2008.

10. Johnson AH, Frierson HF, Zaika A, et al: Expression of tight-junction protein claudin-7 is an early event in gastric tumorigenesis. Am J Pathol 167: 577-584, 2005.

11. Tassi RA, Bignotti E, Falchetti M, et al: Claudin-7 expression in human epithelial ovarian cancer. Int J Gynecol Cancer 18: 1262-1271, 2008.

12. Sobel G, Paska C, Szabo I, Kiss A, Kadar A and Schaff Z: Increased expression of claudins in cervical squamous intraepithelial neoplasia and invasive carcinoma. Hum Pathol 36: 162-169, 2005.

13. Bello IO, Vilen ST, Niinimaa A, Kantola S, Soini Y and Salo T: Expression of claudins 1, 4, 5 and 7 and occludin, and relationship with prognosis in squamous cell carcinoma of the tongue. Hum Pathol 39: 1212-1220, 2008.

14. Moldvay J, Jackel M, Paska C, Soltesz I, Schaff Z and Kiss A: Distinct claudin expression profile in histologic subtypes of lung cancer. Lung Cancer 57: 159-167, 2007.

15. Soini Y: Expression of claudins 1, 2, 3, 4, 5 and 7 in various types of tumours. Histopathology 46: 551-560, 2005. 